\title{
The Year in Science and Medicine
}

\author{
Ze'ev Hochberg and Jean-Claude Carel
}

\section{Review: underestimation of fathers}

\section{Paternal contribution: new insights and future challenges}

\section{Krawetz SA}

Department of Obstetrics and Gynecology, Center for Molecular Medicine and Genetics, Institute for Scientific Computing, Wayne State University School of Medicine, Detroit, Mich., USA

steve@compbio.med.wayne.edu

Nat Rev Genet 2005;6:633-642

Recent progress: Studies on biological processes such as sperm maturation and fertilization now indicate that the paternal contribution has been underestimated.

Summary: The factors that are actually delivered by the sperm at fertilization and their potential developmental functions are discussed using data from humans and animal models.

Applications: Using sperm RNAs have already emerged in reproductive medicine as markers that are indicative of successful vasectomy. They are also beginning to appear in the forensic sciences and, within the next decade, might appear in the environmental sciences.

\begin{abstract}
We were taught that all that fathers contribute to the next generation is half their genome, whereas mothers provide also mitochondrial DNA. This was challenged for a while, but the maternal-only source of zygote mitochondria has now been confirmed. Far from being mere DNA delivery boys, it is now becoming clear that sperms also ship a complex cargo of RNA and proteins that may be crucial for an embryo's early development. Recent progress in reproductive biology indicates that the paternal contribution has been underestimated. Several groups have now established the presence of RNA in human sperm, where they are packaged in the round spermatid, covered with a protective protein coating during the final burst of transcription. Sperm transcripts represent a defined subset of those observed in testis. Phospholipase C (PLC) is delivered by the sperm at fertilization, and has an integral role in the cortical reaction and the induction of a series of $\mathrm{Ca}^{2+}$ oscillations. Human and other primate sperm also deliver a paternally derived centriole. Apparently, paternally derived factors might also segregate and be required until the activation of the zygotic genome. The recent demonstration that RNAs are delivered on fertilization has raised important questions concerning their potential function. To date, several promising candidates have been identified, including an MRNA that encodes PLC- $\gamma$ and a miRNA that might modulate the IGF receptor-2. Studies of spermatozoal RNAs as diagnostic tools have been successful. They can be used as biomarkers of fertility in the clinic and in forensic science. Other applications are also being explored, including their use as environmental monitoring agents.
\end{abstract}

\section{Food for thought: on mythology and twins}

\section{Epigenetic differences arise during the lifetime of monozygotic twins}

Fraga MF, Ballestar E, Paz MF, Ropero S, Setien F, Ballestar ML, Heine-Suner D, Cigudosa JC, Urioste M, Benitez J, Boix-Chornet M, Sanchez-Aguilera A, Ling C, Carlsson E, Poulsen P, Vaag A, Stephan Z, Spector TD, Wu YZ, Plass C, Esteller M

Epigenetics Laboratory, Spanish National Cancer Centre (CNIO), Madrid, Spain

Proc Natl Acad Sci USA 2005;102:10604-10609

Background: Monozygous twins share a common genotype. However, most monozygotic twin pairs are not identical; several types of phenotypic discordance may be observed, such as differences in susceptibilities 
to disease and a wide range of anthropomorphic features. There are several possible explanations for these observations, but one is the existence of epigenetic differences.

Methods: The study examined the global and locus-specific differences in DNA methylation and histone acetylation of a large cohort of monozygotic twins.

Results: Although twins are epigenetically indistinguishable during the early years of life, older monozygous twins exhibited remarkable differences in their overall content and genomic distribution of 5methylcytosine DNA and histone acetylation, affecting their gene-expression portrait.

Conclusions: An appreciation of epigenetics is missing from our understanding of how different phenotypes can originate from the same genotype.

Though they are joined in the night sky as a constellation, you may recall that the Hero twins who sailed with the Argonauts were quite different. Castor was mortal, while his brother Polydeuces was admitted to the godly congregation on Mount Olympus. After 4500 years, this enigma finds an explanation. The phenotypic discordance between monozygotic twins suggests a role for environmental exposures. We start now to understand a potential molecular basis for monozygotic twin discordance: the genomes of monozygotic twins accumulate epigenetic differences as they age. A screen for loci with methylation differences identified both repetitive sequences and single-copy genes, and profiling of methylation at CpG islands show that older twin pairs had 2.5 times as many methylation differences as younger twin pairs. As it goes with epigenetics, it is not yet known whether these changes occur because of environmental exposures or accumulate naturally, and so we cannot yet identify the ultimate cause of phenotypic discordance. But given the importance of epigenetics in the control of cellular distinctiveness, a role for epigenetics in the determination of mortality and the godly congregation seems plausible.

\section{A new receptor family: a gas detection system}

\section{The Drosophila nuclear receptor e75 contains heme and is gas responsive}

Reinking J, Lam MM, Pardee K, Sampson HM, Liu S, Yang P, Williams S, White W, Lajoie G, Edwards A, Krause HM Banting and Best Department of Medical Research, Charles H. Best Institute, Toronto, Ont., Canada Cell 2005; 122:195-207

Background: Nuclear receptors are a family of transcription factors with structurally conserved ligandbinding domains that regulate their activity. Despite intensive efforts to identify ligands, most nuclear receptors are still 'orphans'.

Results: The ligand-binding pocket of the Drosophila nuclear receptor E75 contains a heme prosthetic group. E75 absorption spectra, resistance to denaturants, and effects of site-directed mutagenesis indicate a single, coordinately bound heme molecule. A correlation between the levels of E75 expression and the levels of available heme suggest a possible role as a heme sensor. The oxidation state of the heme iron also determines whether E75 can interact with its heterodimer partner DHR3, suggesting an additional role as a redox sensor. Further, the E75-DHR3 interaction is also regulated by the binding of $\mathrm{NO}$ or $\mathrm{CO}$ to the heme center.

Conclusions: E75 may function as a diatomic gas sensor. Possible mechanisms and roles for these interactions are discussed.

To be on the safe side, we all need gas-detecting systems. It turns out that the fruit fly that helps to rot our garden fruits has such a detector, and that it has a form of endocrine receptor, adopting nitric oxide (NO) and carbon monoxide (CO) as full-fledged hormones. Other than the steroid and thyroid hormone receptors, the ligands that nuclear receptors bind are known in only a few cases, the others being called 'orphan receptors'. We now learn that one such orphan, the Drosophila receptor E75, just found his parents, and that they are quite unusual; they are gases as simple as NO and CO, which are short-lived signaling molecules important in many physiological functions. The receptor includes an iron-containing heme molecule within its ligand-binding pocket. The state of oxidation of the heme affects the ability of the E75 receptor to bind the gases. A number of possible links also exist 
between E75 function and a role in regulating circadian rhythms. NO, CO, and heme appear to be important regulators of circadian oscillators. In vertebrates, 'light' and 'dark' inputs are thought to be converted to signals of $\mathrm{NO}$ and $\mathrm{CO}$, which in turn modulate the circadian cycle. Meanwhile, another orphan receptor, Rev-Erb $\alpha$, may also bind heme and respond to diatomic gases. It is also possible that other nuclear receptors may have adopted or conserved the capacity to bind heme and diatomic gases and to regulate corresponding developmental and physiological functions.

\section{New hope: a premium battery}

\section{Mammalian sprouty proteins assemble into large monodisperse particles having the properties of intracellular nanobatteries}

Wu X, Alexander PB, He Y, Kikkawa M, Vogel PD, McKnight SL

Department of Biochemistry and Cell Biology, University of Texas Southwestern Medical Center, Dallas, Tex., USA

Proc Natl Acad Sci USA 2005;102:14058-14062

Background: Sprouty proteins act as intracellular inhibitors of receptor tyrosine kinase signaling.

Results: The mammalian Sprouty2 protein contains an iron-sulfur complex that can exist in an oxidized, reduced, or nitrosylated state. Purified Sprouty2 assembles into large monodisperse spheres containing approximately 24 polypeptides per particle. Biochemical experiments indicate that the charge state of the iron within Sprouty2 particles may be insulated from ambient intracellular redox.

Conclusions: These features offer the possibility that Sprouty2 particles are capable of receiving, maintaining, and dissipating electrical charge in a manner formally equivalent to a battery.

We first learnt of biological engines, and now of a biological battery; one wonders what comes next. These proteins are called sprouty because a Drosophila with mutant forms of it have excessively branched trachea, and they are thought to control development by interrupting a cell-signaling pathway that regulates growth factors. This paper reports that sulfur atoms in the protein bind to iron, forming a complex that can hold and release electrons. The proteins clump into spherical particles $4-5 \mathrm{~nm}$ across, which might help to insulate the charge, just like a battery does. This may develop into a battery that stores electrical charge out of a protein that is expressed in the cells of mammals and other organisms.

\section{Important finding for future clinical practice: three doors down}

\section{An aneuploid mouse strain carrying human chromosome 21 with Down syndrome phenotypes}

O'Doherty A, Ruf S, Mulligan C, Hildreth V, Errington ML, Cooke S, Sesay A, Modino S, Vanes L, Hernandez D, Linehan JM, Sharpe PT, Brandner S, Bliss TV, Henderson DJ, Nizetic D, Tybulewicz VL, Fisher EM

Department of Neurodegenerative Disease, Institute of Neurology, London, UK

Science 2005;309:2033-2037

Background: Aneuploidies are common chromosomal defects that result in growth and developmental deficits and high levels of lethality in humans.

Method: To gain insight into the biology of aneuploidies, these investigators manipulated mouse embryonic stem cells and generated a trans-species aneuploid mouse line that stably transmits a freely segregating, almost complete human chromosome 21.

Results: This 'transchromosomic' mouse line, Tc1, is a model of trisomy 21, which manifests as Down syndrome (DS) in humans, and has phenotypic alterations in behavior, synaptic plasticity, cerebellar neuronal number, heart development, and mandible size that relate to human DS. 
Conclusions: Transchromosomic mouse lines such as Tc1 may represent useful genetic tools for dissecting other human aneuploidies.

The mechanism of how Down's syndrome (DS) results from three copies of chromosome 21 genes is still enigmatic. We finally have a most accurate mouse model of this condition. The authors did this by injecting mouse embryonic stem cells with copies of the human chromosome. The new mice show characteristics of DS, including behavioral changes and heart defects. From our perspective, we would like to know which genes of that chromosome arrest growth when expressed in three copies, and which will result in the unique thyroid defect of DS that we are yet to work out. For more on the DS thyroid, see Heiko Krude's chapter 'Thyroid: Physiology and Disease' [pp. 19-32]. The method by itself deserves special attention, and will open ways to figure out other human aneuploidies.

\section{A new hormone receptor: take this hormone or leaf it}

\section{Gibberellin insensitive dwarf1 encodes a soluble receptor for gibberellin}

Ueguchi-Tanaka M, Ashikari M, Nakajima M, Itoh H, Katoh E, Kobayashi M, Chow TY, Hsing Yl, Kitano H, Yamaguchi I, Matsuoka M

Bioscience and Biotechnology Center, Nagoya University, Nagoya Japan

Nature 2005;437:693-698

Introduction: Gibberellins (GAs) are phytohormones that are essential for many developmental processes in plants. It has been postulated that plants have both membrane-bound and soluble GA receptors; however, no GA receptors have yet been identified.

Results: This is a report of isolation and characterization of a new GA-insensitive dwarf mutant of rice, gid1. The GID1 gene encodes an unknown protein with similarity to the hormone-sensitive lipases. GID1-green fluorescent protein (GFP) signal was observed preferentially localized in nuclei. Recombinant glutathione $S$-transferase (GST)-GID1 had a high affinity only for biologically active GAs, whereas mutated GST-GID1 corresponding to three gid1 alleles had no GA-binding affinity. The dissociation constant for $\mathrm{GA}_{4}$ was estimated to be around $10^{-7} \mathrm{M}$, enough to account for the GA dependency of shoot elongation. Moreover, GID1 bound to SLR1, a rice DELLA protein, in a GA-dependent manner in yeast cells. GID1 overexpression resulted in a GA-hypersensitive phenotype.

Conclusions: GID1 is a soluble receptor mediating GA signaling in rice.

You can carry on endocrine ideas while gardening. The phyto growth hormone gibberellin is essential for plants' developmental processes such as stem elongation, germination and flower formation. I enjoyed learning that Mendel's dwarf pea is the product of a gibberellin mutation. Now with the isolation and characterization of a new gibberellin-insensitive dwarf mutant of rice, GID1, we have a candidate receptor. GID1 has properties that mark it out as the first known soluble plant hormone receptor, and rice farmers have their own gibberellin insensitivity dwarfism. If they learn as much about plant growth and development as we learned from GHIS, they will understand fundamental stem elongation processes in plants, and crop-selection programs should benefit. Unlike human heightism, plant breeders often doubled grain production by selecting for semi-dwarf varieties of wheat and rice, which led to the 20th century 'green revolution'. 
We now think that RNA came before DNA did, but that does not mean that we know it all about the former. Here is a new chapter in the molecular biology textbook.

\section{A new RNA: stress buster}

\section{Regulating gene expression through RNA nuclear retention}

Prasanth KV, Prasanth SG, Xuan Z, Hearn S, Freier SM, Bennett CF, Zhang MQ, Spector DL Cold Spring Harbor Laboratory, Cold Spring Harbor, N.Y., USA

Cell 2005;123:249-263

Background: Multiple mechanisms have evolved to regulate the eukaryotic genome.

Results: This paper reports CTN-RNA, a mouse tissue-specific approximately 8 -kb nuclear-retained poly $(\mathrm{A})+$ RNA that regulates the level of its protein-coding partner. CTN-RNA is transcribed from the protein-coding mouse cationic amino acid transporter 2 (mCAT2) gene through alternative promoter and poly(A) site usage. CTN-RNA is diffusely distributed in nuclei and is also localized to paraspeckles. The 3'-UTR of CTN-RNA contains elements for adenosine-to-inosine editing, involved in its nuclear retention. Interestingly, knockdown of CTN-RNA also downregulates mCAT2 mRNA. Under stress, CTN-RNA is posttranscriptionally cleaved to produce protein-coding mCAT2 mRNA.

Conclusions: These findings reveal a role of the cell nucleus in harboring RNA molecules that are not immediately needed to produce proteins but whose cytoplasmic presence is rapidly required upon physiologic stress. This mechanism of action highlights an important paradigm for the role of a nuclearretained stable RNA transcript in regulating gene expression.

Transcription factors, like some of the steroid hormone receptors, reside in the cytoplasm and are then rapidly imported into the nucleus upon demand by a cellular signal to initiate transcription. We now learn of an analogous mechanism that involves RNA. 'The central dogma' of molecular biology holds that genetic information normally flows from DNA to RNA to protein. As a consequence, it has been assumed that genes generally encode for proteins and that proteins fulfill not only structural and catalytic but also most regulatory functions in cells. However, a subset of RNAs executes functions without being translated into protein. Such RNAs include housekeeping ribosomal RNA, transfer RNA, and Urich small nuclear and nucleolar RNAs, as well as nuclear-retained RNAs that execute regulatory roles in various aspects of gene expression. Interestingly, the percentage of non-coding DNA in prokaryotes is $<25 \%$ of the total genome compared to about $98 \%$ in humans. This paper reports a new class of messenger RNA. Some RNA transcripts of a mouse gene called mCAT2 remain as back-up copies in the cell nucleus, only being released into the cytoplasm when the cell is stressed. The normal protein product of mCAT2 helps cells to respond to pressures such as viral infection; so mobilizing this reserve RNA might allow the cell to react more quickly. The rapid response mechanism of nuclear RNA release for protein synthesis may be a general paradigm for the production of some critical regulatory proteins. 


\section{Nuclear reprogramming of somatic cells after fusion with human embryonic stem cells}

Cowan CA, Atienza J, Melton DA, Eggan K

Howard Hughes Medical Institute, Harvard Stem Cell Institute, Department of Molecular and Cellular Biology,

Harvard University, Cambridge, Mass., USA

Science 2005;309:1369-1373

Background: This paper explored the use of embryonic stem cells as an alternative to oocytes for reprogramming human somatic nuclei.

Methods: Human embryonic stem (hES) cells were fused with human fibroblasts, resulting in hybrid cells that maintain a stable tetraploid DNA content and have morphology, growth rate, and antigen expression patterns characteristic of hES cells.

Results: Differentiation of hybrid cells in vitro and in vivo yielded cell types from each embryonic germ layer. Analysis of genome-wide transcriptional activity, reporter gene activation, allele-specific gene expression, and DNA methylation showed that the somatic genome was reprogrammed to an embryonic state.

Conclusions: hES cells can reprogram the transcriptional state of somatic nuclei and provide a system for investigating the underlying mechanisms.

The prospect of regenerating the entire range of varied cell types from an adult somatic cell is an appealing thought. Yet, development of fertilized egg involves progressive loss of pluripotency as cells differentiate, and essentially, there is no way back for differentiated cells. In contrast, embryonic stem cells (ESC) from the blastocyst stage are pluripotent and retain the capacity to differentiate into all cell types. As scientists venture to confer this unique property from ESC directly to adult somatic cells, this report suggest that it may be possible, and the process involved is called nuclear reprogramming. It involves the erasing and resetting of epigenetic modifications to the DNA sequence, principally methylation of CpG dinucleotides. By fusing ESC with fibroblasts, this new report reveals that the human fibroblast nucleus was indeed reprogrammed and became pluripotent, while the expression of fibroblast-specific genes was repressed. OCT4, a key pluripotency-specific gene that is repressed in mature fibroblasts with a methylated promoter region, became unmethylated, a necessary step for reinitiation of its expression. This is a heritable epigenetic modification, which suggests that the effects may not be just transient but could carry on subsequently. The efficiency of the fusion process between hESC and somatic cells is still very low and requires the use of drugs to select for productive hybrids from a pool of unfused cells. That hESC have the potential to reprogram somatic cell nuclei is a big step forward.

\section{All in the family}

The journal Science elected evolution as the field that produced the greatest breakthroughs of the year 2005. Here is a small selection.

\section{Initial sequence of the chimpanzee genome and comparison with the human genome}

Chimpanzee Sequencing and Analysis Consortium

Nature 2005;437:69-87

Method: Through comparison with the human genome, this team generated a largely complete catalogue of the genetic differences that have accumulated since the human and chimpanzee species diverged from our common ancestor, constituting approximately 35 million single-nucleotide changes, 5 million insertion/deletion events, and various chromosomal rearrangements. They use this catalogue to explore the magnitude and regional variation of mutational forces shaping these two genomes, and the strength of positive and negative selection acting on their genes. 
Results: In particular, they find that the patterns of evolution in human and chimpanzee protein-coding genes are highly correlated and dominated by the fixation of neutral and slightly deleterious alleles. Conclusion: The chimpanzee genome can be used as an out-group to investigate human population genetics and identify signatures of selective sweeps in recent human evolution.

As our closest living evolutionary relatives, chimpanzees are especially suited to teach us about ourselves. Somewhere in this register of divergence lies the genetic draft for the traits that make us human: upright gait, sparse body hair, a big and creative brain. The genome data confirm our close kinship with the chimp; we differ by only about $1 \%$ in the aligned nucleotide bases, and the average protein differs by less than two amino acids. But a surprisingly large chunk of non-coding material is either inserted or deleted in the chimp as compared to the human, bringing the total difference in DNA between our two species to about $4 \%$. With the human genome already in hand, researchers line up chimp and human DNA and will examine one by one the 40 million evolutionary events that separate them from us. More than 50 genes present in the human genome are missing or partially deleted from the chimp genome. Six regions in the human genome have strong signatures of selective sweeps over the past 250,000 years. One region contains more than 50 genes, while another contains no known genes and lies in an area that are refer to as a 'gene desert'. Intriguingly, this gene desert may contain elements regulating the expression of the nearby protocadherin gene, which has been implicated in patterning of the nervous system, including the hypothalamus. The genomic analyses also show that humans and chimps have accumulated more potentially deleterious mutations in their genomes over the course of evolution than have mice, rats and other rodents. While such mutations can cause diseases that may wear away a species' overall fitness, they may have also made primates more adaptable to rapid environmental changes, and enabled them to achieve unique evolutionary adaptations. The chimpanzee genome sequence will have a major impact on our understanding of human disease, human evolution, and human population genetics. Just as a small hint to its enormous importance: humans are highly susceptible to AIDS, coronary heart disease, chronic viral hepatitis, and malignant malarial infections; chimps aren't. But chimps are important beyond their contribution to humans. Like us, they are self-aware; they have tools, medicines, cultures and even politics. Not only do they communicate with each other, they can learn to use sign language and have conversations with people. For every chimp there are 20,000 people on earth, and if we don't stop eliminating their habitat, and that of other big apes, we will soon be left here alone with our politics, which is by far more devastating.

\section{New paradigms: pardon the expression}

\section{Expression profiling in primates reveals a rapid evolution of human transcription factors}

Gilad Y, Oshlack A, Smyth GK, Speed TP, White KP

Department of Genetics, Yale University, New Haven, Conn., USA

gilad@uchicago.edu

Nature 2006;440:242-245

Background: Although it has been hypothesized for 30 years that many human adaptations are likely to be due to changes in gene regulation, almost nothing is known about the modes of natural selection acting on regulation in primates.

Results: Here the authors identify a set of genes for which expression is evolving under natural selection. A new multi-species complementary DNA array is used to compare steady-state messenger RNA levels in liver tissues within and between humans, chimpanzees, orangutans and rhesus macaques. Using estimates from a linear mixed model, a set of genes is identified for which expression levels have remained constant across the entire phylogeny ( $\sim 70$ million years), and are therefore likely to be under stabilizing selection. Among the top candidates are five genes with expression levels that have previously been shown to be altered in liver carcinoma. A number of genes with similar expression levels among nonhuman primates but significantly elevated or reduced expression in the human lineage are identified, 
features that point to the action of directional selection. Among the gene set with a human-specific increase in expression, there is an excess of transcription factors; the same is not true for genes with increased expression in chimpanzee.

Evolutionary studies tend to focus on alterations in genes and proteins. But evolutionary change can often occur through modified gene expression, the major determining factor of protein abundance in the cell. Adaptation over a short evolutionary time may often proceed by modifications in the regulation and interaction of genes rather than in the protein gene products themselves. This paper describes a study of gene expression in four primate species, to identify similarities and differences in gene expression between humans, chimpanzees, orangutans and rhesus. They demonstrate that most genes are under natural selection to maintain a constant level of expression, but that a few genes show evidence of species-specific changes. Some groups of genes, particularly those encoding gene-transcription factors, tend to include greater numbers of upregulated genes in humans. Genes that are significantly up- or downregulated in humans, compared with other species, are often genes that have changed rapidly at the DNA-sequence level; so there seems to be a correspondence between genes with altered expression and genes that have been targeted by positive Darwinian selection in their protein-coding regions. This makes sense; we would expect changes in the function of a protein to be followed by changes in its distribution and abundance. Likewise, we may expect genes that have suffered a loss or reduction in functionality to subsequently experience an increased rate of evolution in both the sequence of the protein it encodes and its expression level, because selective constraints on it will have been relaxed.

\section{Of precious junk and biological complexity}

\section{Adaptive evolution of non-coding DNA in Drosophila}

Andolfatto $P$

Section of Ecology, Behavior and Evolution, Division of Biological Sciences, University of California San Diego,

La Jolla, Calif., USA

pandolfatto@ucsd.edu

Nature 2005;437:1149-1152

Background: A large fraction of eukaryotic genomes consists of DNA that is not translated into protein sequence, and little is known about its functional significance.

Results: Several classes of non-coding DNA in Drosophila are evolving considerably slower than synonymous sites, and yet show an excess of between-species divergence relative to polymorphism when compared with synonymous sites. The former is a hallmark of selective constraint, but the latter is a signature of adaptive evolution, resembling general patterns of protein evolution in Drosophila. The author estimates that about $40-70 \%$ of nucleotides in intergenic regions, untranslated portions of mature mRNAs (UTRs) and most intronic DNA are evolutionarily constrained relative to synonymous sites. A substantial fraction of the nucleotide divergence in these regions was driven to fixation by positive selection (about $20 \%$ for most intronic and intergenic DNA, and $60 \%$ for UTRs).

Conclusions: A large fraction of the non-translated genome is functionally important and subject to both purifying selection and adaptive evolution.

Implications: Although positive selection is clearly an important facet of protein evolution, adaptive changes to non-coding DNA might have been considerably more common in the evolution of D. melanogaster.

Once designated 'junk DNA', non-coding DNA has now its own website and unique name - ncDNA. This paper suggests that the amount of ncDNA per genome ( $98 \%$ in human) is a more accurate indicator of biological complexity than either gene number or genome size. It is therefore highly likely that these sequences are functional, and the idea of 'junk' may need to be trashed. It is now possible that introns, intergenic sequences, repeat elements, and genomic DNA previously regarded as genetically inert may be far more important to the evolution and functional repertoire of complex organisms than has been previously appreciated. 


\title{
Characterization of the reconstructed 1918 Spanish influenza pandemic virus
}

\author{
Tumpey TM, Basler CF, Aguilar PV, Zeng H, Solorzano A, Swayne DE, Cox NJ, Katz JM, Taubenberger JK, Palese P, \\ Garcia-Sastre A \\ Influenza Branch, Division of Viral and Rickettsial Diseases, National Center for Infectious Diseases, Centers for \\ Disease Control and Prevention, Atlanta, Ga., USA \\ tft9@cdc.gov
}

Science $2005 ; 310: 77-80$

Background: The pandemic influenza virus of 1918-1919 killed an estimated 20-50 million people worldwide.

Methods: With the recent availability of the complete 1918 influenza virus coding sequence, these investigators used reverse genetics to generate an influenza virus bearing all eight gene segments of the pandemic virus to study the properties associated with its extraordinary virulence.

Results: In stark contrast to contemporary human influenza H1N1 viruses, the 1918 pandemic virus had the ability to replicate in the absence of trypsin, caused death in mice and embryonated chicken eggs, and displayed a high-growth phenotype in human bronchial epithelial cells.

Conclusions: The coordinated expression of the 1918 virus genes most certainly confers the unique highvirulence phenotype observed with this pandemic virus.

\begin{abstract}
Avian influenza has become this year's most urgent biomedical threat. Using tissue from a body that had been frozen in the Alaskan permafrost for almost a century, another group of investigators sequenced the three unknown genes from the 1918 flu virus, which caused the epidemic that killed 20-50 million people. Mostly, fatal flu strains surface when an animal virus combines with an existing human virus. But after studying the genetic data, they concluded that the 1918 virus started out as a pure avian strain, and a handful of mutations had enabled it to easily infect human hosts. The possible evolution of such an infectious ability in the 2006 bird flu is why we all worry about a new pandemic. This paper reports that the 1918 strain had lost its dependence on trypsin, which viruses typically make use out of their hosts as they infect cells. Instead, the 1918 strain depended on an inhouse enzyme. As a result, the reconstructed virus reached exceptionally high concentrations in the lung tissue of mice tested, helping explain its virulence. The finding could spot new ways to prevent similar fatal infections should the current event go virulent to human. In Mozambique they say that that Only God can help the tail-less cattle chase away the bugs that trouble them. Similar research efforts are more likely to chase away that threatening new avian bug.
\end{abstract}

\section{Systems biology: signal ahead of a junction}

\section{A systems model of signaling identifies a molecular basis set for cytokine-induced apoptosis}

Janes KA, Albeck JG, Gaudet S, Sorger PK, Lauffenburger DA, Yaffe MB

Biological Engineering Division, Department of Biology, Massachusetts Institute of Technology, Cambridge, Mass., USA Science 2005;310:1646-1653

Background: Signal transduction pathways control cellular responses to stimuli, but it is unclear how molecular information is processed as a network.

Methods: These investigators constructed a systems model of 7,980 intracellular signaling events that directly links measurements to 1,440 response outputs associated with apoptosis.

Results: The model accurately predicted multiple time-dependent apoptotic responses induced by a combination of the death-inducing cytokine tumor necrosis factor with the prosurvival factors epidermal growth factor and insulin. By capturing the role of unsuspected autocrine circuits, activated by transforming growth factor- $\alpha$ and interleukin- $1 \alpha$, the model revealed new molecular mechanisms connecting signaling to 
apoptosis. The model derived two groupings of intracellular signals that constitute fundamental dimensions (molecular 'basis axes') within the apoptotic signaling network. Projection along these axes captures the entire measured apoptotic network.

Conclusion: Cell survival is determined by signaling through this canonical basis set.

Systems biologists are busy trying to understand how cells respond to the innumerable chemicals and signals showering them from all sides. For more, see Roland Pfäffle's chapter 'Systems Biology' [pp. 77-86]. Complex biological systems are best modeled as highly modular, flowing systems exhibiting a plasticity that allows them to adapt to a vast array of conditions. The tools of molecular biology and data mining allow now for a network-oriented view of biological systems and for explaining the underlying causes of disease, as well as the best ways to target disease. Ultimately, this will help to ensure that the right drug is administered to the right patient at the right time. Along the way, this group discovered new apoptosis-signaling routes. Other fruits of the year in systems biology are 40 new genes that help trigger obesity, three of which had never been identified before and novel insights into signaling networks that control T cells and CA1 neurons in the hippocampus. Keep an open mind for systems biology and pediatric endocrinology, which will be the focus of a meeting organized by ESPE for 2008.

\section{New mechanism. Is your $X$ active?}

\section{Regulation of X-chromosome counting by Tsix and Xite sequences}

Lee JT

Howard Hughes Medical Institute, Department of Molecular Biology, Massachusetts General Hospital, Department of Genetics, Harvard Medical School, Boston, Mass., USA

lee@molbio.mgh.harvard.edu

Science 2005;309:768-771

Background: In mammals, X-inactivation establishes X-chromosome dosage parity between males and females. How X-chromosome counting regulates this process remains elusive, because neither the hypothesized inactivation 'blocking factor' nor the required cis-elements have been defined.

Results: Here, a mouse knockout and transgenic analysis identified DNA sequences within the non-coding Tsix and Xite genes as numerators. Homozygous deficiency of Tsix resulted in 'chaotic choice' and a variable number of inactive X's, whereas overdosage of Tsix/Xite inhibited X-inactivation. Thus, counting was affected by specific Tsix/Xite mutations, suggesting that counting is genetically separable from but molecularly coupled to choice. The mutations affect XX and XY cells differently, demonstrating that counting and choice are regulated not by one 'blocking factor', but by both a 'blocking' and a 'competence' factor.

\section{Pair to part}

\section{Transient homologous chromosome pairing marks the onset of $X$ inactivation}

Xu N, Tsai CL, Lee JT

Howard Hughes Medical Institute, Department of Molecular Biology, Massachusetts General Hospital, Department of Genetics, Harvard Medical School Boston, Mass., USA

Science 2006;311:1149-1152

Background: Mammalian X inactivation turns off one female X chromosome to enact dosage compensation between XX and XY individuals. $\mathrm{X}$ inactivation is known to be regulated in cis by Xite, Tsix, and Xist, but in principle the two Xs must also be regulated in trans to ensure mutually exclusive silencing. 
Results: Interchromosomal pairing mediates this communication. Pairing occurs transiently at the onset of $\mathrm{X}$ inactivation and is specific to the $\mathrm{X}$-inactivation center. Deleting Xite and Tsix perturbs pairing and counting/choice, whereas their autosomal insertion induces de novo X-autosome pairing. Ectopic $\mathrm{X}$-autosome interactions inhibit endogenous X-X pairing and block the initiation of X-chromosome inactivation. Thus, Tsix and Xite function both in cis and in trans.

Conclusion: Tsix and Xite regulate counting and mutually exclusive choice through X-X pairing.

In mammals, most genes on one $X$ chromosome are inactivated in females to equalize the dose of $\mathrm{X}$ chromosome genes between $\mathrm{XX}$ and $\mathrm{XY}$ individuals. This regulatory process, known as $\mathrm{X}$ chromosome inactivation [1], results in transcription of the Xist gene, which encodes a structural RNA that coats the inactive $X$ chromosome and is essential to the inactivation process. One of the key questions regarding $X$ inactivation is how $X$ chromosomes are counted (in order not to inactivate the $X$ chromosome of $X Y$ individuals for instance) and how the chromosome is chosen for inactivation. The Xist gene is part of the $X$ inactivation center, together with the Tsix gene, its antisense partner, and the Xite gene, an upstream regulator. These two rather complex papers shed light on the mechanisms involved in $\mathrm{X}$ chromosome counting and pairing during the inactivation process. In the first (singleauthored!) paper, the author has used embryonic (ES) cell lines from mice with mutations in the $X$ inactivation center genes to assess their consequences on $X$-chromosome counting. ES cells from XX mice with a homozygous mutation in Tsix were grown into embryoid bodies. These embryoid bodies grew poorly and displayed a chaotic pattern of $X$ inactivation (cells with 2 active, 1 active or 0 active $\mathrm{X}$ chromosome). In contrast, transgenic expression of Tsix or Xite (with transgenes inserted in autosomes) led to inactivation of the autosome but resulted in failure to initiate $X$ chromosome inactivation. Therefore, loss of function of Tsix and overdosing of Tsix or Xite led to opposite phenotypes and the author proposes that the DNA sequence between Tsix and Xite functions as a numerator for $X$ chromosomes. She proposes a two-factor model to explain the maintenance of normal autosome/ $X$ ratio in accordance with the experimental findings.

In the second paper from the same laboratory (not alone this time), they analyze how the system chooses only one $X$ chromosome at a time for inactivation. They show that at onset of $X$ inactivation the two $X$ chromosomes get in close contact and that $X$ pairing occurs just before or during Xist upregulation. The two $X$ pair homologously at the onset of $X$ inactivation with Tsix and Xite as nucleation centers. This generates a crosstalk resulting in asymmetric marking of one of the $X$ chromosomes. This chromosome crosstalk adds a new dimension to complex regulatory events at the initial stages of random $\mathrm{X}$ inactivation. In addition, pairing is not unique to this process and is probably a common mechanism for gene regulatory processes. This will likely become a recurrent theme in epigenetic phenomena and it adds to the complexity in understanding the mechanisms underlying sex chromosome abnormalities.

\section{New technology. A human genome sequence for USD 1,000}

\section{Genome sequencing in microfabricated high-density picolitre reactors}

Margulies M, Egholm M, Altman WE, Attiya S, Bader JS, Bemben LA, Berka J, Braverman MS, Chen YJ, Chen Z, Dewell SB, Du L, Fierro JM, Gomes XV, Godwin BC, He W, Helgesen S, Ho CH, Irzyk GP, Jando SC, Alenquer ML, Jarvie TP, Jirage KB, Kim JB, Knight JR, Lanza JR, Leamon JH, Lefkowitz SM, Lei M, Li J, Lohman KL, Lu H, Makhijani VB, McDade KE, McKenna MP, Myers EW, Nickerson E, Nobile JR, Plant R, Puc BP, Ronan MT, Roth GT, Sarkis GJ, Simons JF, Simpson JW, Srinivasan M, Tartaro KR, Tomasz A, Vogt KA, Volkmer GA, Wang SH, Wang Y, Weiner MP, Yu P, Begley RF, Rothberg JM

454 Life Sciences Corp., Branford, Conn., USA

Nature 2005:437:376-380

Background: The proliferation of large-scale DNA-sequencing projects in recent years has driven a search for alternative methods to reduce time and cost.

Results: The authors describe a scalable, highly parallel sequencing system with raw throughput significantly greater than that of state-of-the-art capillary electrophoresis instruments. The apparatus uses a 
novel fibre-optic slide of individual wells and is able to sequence 25 million bases, at $99 \%$ or better accuracy, in one 4-hour run. To achieve an approximately 100-fold increase in throughput over current Sanger sequencing technology, the authors have developed an emulsion method for DNA amplification and an instrument for sequencing by synthesis using a pyrosequencing protocol optimized for solid support and picolitre-scale volumes. They show the utility, throughput, accuracy and robustness of this system by shotgun sequencing and de novo assembly of the Mycoplasma genitalium genome with $96 \%$ coverage at $99.96 \%$ accuracy in one run of the machine.

Have you ever dreamed of ordering a full genome sequence to work out a complex patient? This paper tells that this might probably become feasible relatively soon. In the accompanying commentary [2], Craig Venter comments that although DNA sequencing techniques have markedly improved, it still costs approximately 10-25 million US dollars to sequence a single human genome. The system described here could potentially allow an individual to prepare and sequence an entire genome in a few days. Its complexity lies in DNA sample preparation and in the microfabricated platform that contains 1.6 million reactors containing each 1 picoliter. The system has limitations in that it can read relatively short sequences at a time $(\approx 100 \mathrm{bp})$ so that stitching the results in long sequences might be problematic. The accuracy is not as good as conventional sequencing approaches and only one strand can be read at a time. Although it will take time for this technology to become fully functional and enter the routine, it is certainly a development that we want to hear about. Of course, it will be important to improve data analysis in parallel, since we would be embarrassed to receive a 3,000megabase printout on our hospital desk.

\title{
New concerns. Learned fear and racial groups
}

\section{The role of social groups in the persistence of learned fear}

\author{
Olsson A, Ebert JP, Banaji MR, Phelps EA
}

Department of Psychology, New York University, New York, N.Y., USA

Science 2005;309:785-787

Background: Classical fear conditioning investigates how animals learn to associate environmental stimuli with an aversive event.

Methods: The authors examined how the mechanisms of fear conditioning apply when humans learn to associate social ingroup and outgroup members with a fearful event, with the goal of advancing our understanding of basic learning theory and social group interaction. Primates more readily associate stimuli from certain fear-relevant natural categories, such as snakes, with a negative outcome relative to stimuli from fear-irrelevant categories, such as birds. Whether this bias in fear conditioning extends to social groups defined by race was assessed.

Results: The results indicate that individuals from a racial group other than one's own are more readily associated with an aversive stimulus than individuals of one's own race, among both white and black Americans. This prepared fear response might be reduced by close, positive interracial contact.

\section{The primate amygdala represents the positive and negative value of visual stimuli during learning}

Paton JJ, Belova MA, Morrison SE, Salzman CD

Center for Neurobiology and Behavior, Columbia University, Unit 87, New York, N.Y., USA

Nature 2006;439:865-870

Background: Visual stimuli can acquire positive or negative value through their association with rewards and punishments, a process called reinforcement learning. Although we now know a great deal about how the brain analyses visual information, we know little about how visual representations become linked with values.

Methods: Analysis of the amygdala, a brain structure implicated in reinforcement learning. Recording of the activity of individual amygdala neurons in monkeys while abstract images acquired either positive 
or negative value through conditioning. After monkeys had learned the initial associations, the image value assignments were reversed. Neural responses in relation to these reversals were examined in order to estimate the relative contribution to neural activity of the sensory properties of images and their conditioned values.

Results: Changes in the values of images modulate neural activity. This modulation occurs rapidly enough to account for, and correlates with, monkeys' learning. Furthermore, distinct populations of neurons encode the positive and negative values of visual stimuli.

Conclusion: Behavioral and physiological responses to visual stimuli may therefore be based in part on the plastic representation of value provided by the amygdala.

Intergroup conflicts are tragically part of the daily news, if not part of our daily lives. For the general absurdity of these conflicts, there is a scientific approach to try to understand how attitudes between ethnic groups are formed. The authors use fear conditioning as a tool to evaluate the differential response to outgroup vs. ingroup human faces. The classical experiment is to associate a fear-relevant (snake, spider) or fear-irrelevant (bird, butterfly) image to a mild electric shock. The image is then presented again without the electric shock (extinction phase) and skin conductance is measured. Learned fear is measured by the persistence of a fearful reaction to the image vs. the relevant control. In this example, in both humans and primates, conditioned fear response persists to spiders but not to birds. Here, the authors performed the same experiments presenting pictures of black or white males with neutral facial expression to black or white American participants. They find that during the extinction phase, fearful reactions to racial outgroup faces persist, while those to racial ingroup disappear. In factorial analysis, this reaction was unrelated to racial attitudes and beliefs or amount of contact with outgroup members. The only factor identified was interracial dating, associated with attenuated outgroup conditioned fear. The lengthy discussion that follows tries to weigh the respective influences of evolutionary (atavistic) reactions vs. social learning, but this appears to be essentially impossible. Fear conditioning results automatically from pairing a neutral stimulus with an aversive one and does not require conscious mediation. The findings show that these non-conscious processes can be counteracted by interracial exposure and contact, and help combat the development of such stereotypes. They also confirm that no information or image is never neutral and make us think that the development of visual news media in the second half of the 20th century might have contributed to reinforce interracial aversion.

The amygdala is a collection of nuclei located deep in the medial temporal lobe receiving visual and other sensory systems inputs. The amygdala also receives signals that represent stimuli in relation to expectations. Brain imaging studies have shown that fear conditioning correlates with activity in the amygdala. The second paper examines amygdala function in primates during learning. The animals were shown images associated with a positive signal (sweet reward), a negative signal (aversive airpuff in the face) or nothing, and learned the association (for instance they blinked in anticipation of the air-puff when they saw the negative images). Then the image associations were reversed and the time taken by the monkey to learn the new association was measured and correlated with single neuron activity in the amygdala. The data show that neurons begin to change their activity within a few trials of change in image value, and that the change in activity tracks behavioral learning. This type of learning (called reinforcement learning) is thought to provide a model to study emotions and the responses to sensory stimuli endowed with a positive or negative value. How the amygdala neurons interconnect with other brain areas remains to be determined. Last year we learnt that amygdala damage resulted in impaired fear recognition [3]. This year, amygdala function is once again at the center of non-conscious mental activities that involve processing sensory signals. These processes have certainly been essential in species survival and are also part of daily social interactions in the modern world. 


\section{Intellectual ability and cortical development in children and adolescents}

Shaw P, Greenstein D, Lerch J, Clasen L, Lenroot R, Gogtay N, Evans A, Rapoport J, Giedd J

Child Psychiatry Branch, National Institute of Mental Health, Bethesda, Md., USA

shawp@mail.nih.gov

Nature 2006;440:676-679

Background: Children who are adept at any one of the three academic 'Rs' (reading, writing and arithmetic) tend to be good at the others, and grow into adults who are similarly skilled at diverse intellectually demanding activities. Determining the neuroanatomical correlates of this relatively stable individual trait of general intelligence has proven difficult, particularly in the rapidly developing brains of children and adolescents.

Results: The trajectory of change in the thickness of the cerebral cortex, rather than cortical thickness itself, is most closely related to level of intelligence. Using a longitudinal design, the authors find a marked developmental shift from a predominantly negative correlation between intelligence and cortical thickness in early childhood to a positive correlation in late childhood and beyond. Additionally, level of intelligence is associated with the trajectory of cortical development, primarily in frontal regions implicated in the maturation of intelligent activity. More intelligent children demonstrate a particularly plastic cortex, with an initial accelerated and prolonged phase of cortical increase, which yields to equally vigorous cortical thinning by early adolescence.

Conclusion: The study indicates that the neuroanatomical expression of intelligence in children is dynamic.

In 1954 the Canadian neurobiologist Wilder Penfield (1891-1976) wrote: How it may be that ganglionic activity is transformed into thinking, and how it is that thought is converted into neuronal activity of conscious voluntary action, we have no knowledge. Fifty years later, and we still know little about mind and body and how anatomy, chemistry and physiology are transformed into poetry or music. Relating the size or shape of the brain to intelligence reminds us of 19th century scientists who hoped to unravel the secrets of cognition through neuroanatomical correlates. However, this paper has taken a dynamic approach to the question by longitudinally examining brain development by MRI in a group of 307 control subjects from childhood (age 7) to adulthood (age 19). They found virtually no correlation between IQ and cortical thickness but found that the dynamics of cortical thickness are highly correlated with IQ. Children with the highest IQs start with a thinner cortex that thickens rapidly to a maximum at age 12 and then thins again. In contrast, those with an average IQ start with a thicker cortex that thins progressively during development. Regional differences are seen and the differing trajectories are more prominent in the prefrontal cortex, whose activation is known to correlate functionally with intelligence. No influence of handedness or gender was observed, and it would have been interesting to observe the influence of pubertal development on these patterns. The basis of this developmental diversity remains to be determined, both in terms of anatomy and of factors, genetic or environmental, influencing it. Anyhow, this paper tells us that dynamics rather than brain size matters. It helps us understand why the child's brain is so sensitive to insults. It is likely that hormonal influences are part of the variability of these processes and this will certainly be important to pediatric endocrinologists in the future. 


\section{Effects of early childhood psychosocial stimulation and nutritional supplementation on cognition and education in growth-stunted Jamaican children: prospective cohort study}

Walker SP, Chang SM, Powell CA, Grantham-McGregor SM

Epidemiology Research Unit, Tropical Medicine Research Institute, University of the West Indies, Mona, Jamaica susan.walker@uwimona.edu.jm

Lancet 2005;366:1804-1807

Background: Growth retardation affects about a third of children younger than age 5 years in developing countries and is associated with poor development. Previously, the authors did a trial of nutritional supplementation and psychosocial stimulation in stunted children aged 9-24 months. Non-stunted children were also assessed. Both types of intervention improved development.

Results: The effects of early interventions on cognition and education in 103 of the 129 stunted children are compared with outcome in 64 of the 84 non-stunted children now aged 17-18 years. No significant effects of nutritional supplementation were recorded. Compared with no intervention, stimulation resulted in higher full scale IQ scores (coefficient $0.38, \mathrm{p}=0.02$ ) and higher scores on the verbal subscale $(0.37, \mathrm{p}=0.02)$, Peabody picture vocabulary test $(7.84, \mathrm{p}=0.03)$, verbal analogies $(0.26$, $\mathrm{p}=0.03)$, and reading tests $(4.73, \mathrm{p}=0.007$, and $2.7, \mathrm{p}=0.001)$. Overall, stunted non-stimulated participants had significantly poorer scores than the non-stunted group on 11 of 12 cognitive and educational tests.

Conclusion: Stunting in early childhood is associated with cognitive and educational deficits in late adolescence, which are reduced by stimulation at a young age.

The investigators of this study are to be praised for its rigorous design and for the long-term followup provided, all in the difficult environment of a developing country. The study was initiated in 1986 and 129 growth-retarded children (called here stunted, height $\leq 2$ SDS), aged 9-24 months, were randomly assigned to receive food, psychosocial stimulation, both or none. Food supplementation consisted in $1 \mathrm{~kg}$ of milk formula per week. Psychosocial stimulation by the family was encouraged through a weekly 1 -hour visit by a community worker. In the short term, all interventions improved growth with a complete catch-up in those receiving both interventions. The children were assessed for cognition and education at age 17-18 years. It is interesting to see that food supplementation had no effect on IQ, cognition, or school performance. In contrast, psychosocial stimulation increased IQ and reading comprehension and was associated with a lower rate of school failure, though the effect sizes were quite modest. These findings are relatively counterintuitive for those of us who thought that nutritional substrates are the \#1 need for brain development. The findings confirm that stunting in the first 2 years of life has long-lasting developmental consequences and that our efforts should be directed towards prevention. They show the possibility to implement a childhood stimulation program in a difficult environment and its highly beneficial effects. The authors rightly discuss that the results could be due to inappropriate food supplementation (shared with other children, not enough in terms of duration or quantity). These findings, although obtained in an extreme environment, might also apply to children growing in a more opulent environment where growth-retarded infants, both born SGA or premature, might also benefit from focused psycho-developmental stimulation. 


\section{Why most published research findings are false}

loannidis JP

Department of Hygiene and Epidemiology, University of Ioannina School of Medicine, loannina, Greece

jioannid@cc.uoi.gr

PLoS Med 2005;2:e124

Background: There is increasing concern that most current published research findings are false. The probability that a research claim is true may depend on study power and bias, the number of other studies on the same question, and, importantly, the ratio of true to no relationships among the relationships probed in each scientific field.

Results: In this framework, a research finding is less likely to be true when the studies conducted in a field are smaller; when effect sizes are smaller; when there is a greater number and lesser preselection of tested relationships; where there is greater flexibility in designs, definitions, outcomes, and analytical modes; when there is greater financial and other interest and prejudice; and when more teams are involved in a scientific field in chase of statistical significance. Simulations show that for most study designs and settings, it is more likely for a research claim to be false than true. Moreover, for many current scientific fields, claimed research findings may often be simply accurate measures of the prevailing bias.

Interpretation: The author discusses the implications of these problems for the conduct and interpretation of research.

The large number of papers published in biomedical peer-reviewed journals is good news, indicating that the field is active and that authors are striving to publish. This matter of fact drove the editors and associate editors of the Yearbook of Pediatric Endocrinology to start the initiative. However, this increasing number of published papers, each of them reporting several positive hits, after having tested an even greater number of hypotheses or associations, raises the question of the proportion of true findings. Here, several concerns on the methodology of contemporary science (the reign of $p<0.05)$ are raised and addressed through mathematical modeling. Several theoretical curves are drawn that plot the probability that a research is true vs. the prestudy odds, power and a ' $u$ ' value, meant to represent the prevailing bias. Back to practical considerations, we get reassuring words that larger studies, higher effect sizes, lower number of hypotheses tested, strict analyses of outcomes, lower competing interests and less competitive fields yield the more reliable (i.e. true) research findings. Potential ways to improve the situation are discussed. In any case, this paper reminds us that everything that is written is not necessarily true and that reading the biomedical literature should be done with a grain of salt. But don't lose your faith in science; it is probably the only discipline that keeps checking itself continuously, as this paper does. James Morrow, who is best known for his Godhead Trilogy wrote: Everybody thinks he's being oh-so-deep when he says science doesn't have all the answers. ... Science does have all the answers ... The problem is that we don't have all the science.

\section{The trouble with inflation}

\section{Three-year Wilkinson Microwave Anisotropy Probe (WMAP)1 observations: beam profiles, data processing, radiometer characterization and systematic error limits}

Jarosik N, Barnes C, Greason MR, Hill RS, Nolta MR, Odegard N, Weiland JL, Bean R, Bennett CL, Dor'e O, Halpern M, Hinshaw G, Kogut A, Komatsu E, Limon M, Meyer SS, Page L, Spergel DN, Tucker GS, Wollack E, Wright EL http://lambda.gsfc.nasa.gov/product/map/current/

Astrophys J 2006; March 16

Introduction: NASA's Wilkinson Microwave Anisotropy Probe (WMAP) satellite has now completed 3 years of observations of the cosmic microwave background radiation. The 3 -year data products include 
several sets of full sky maps of the Stokes I, Q and U parameters in 5 frequency bands, spanning 23-94 GHz, and supporting items, such as beam window functions and noise covariance matrices.

Results: The processing used to produce the current sky maps and supporting products represents a significant advancement over the first year analysis, and provides improvements to the pointing reconstruction, radiometer gain modeling, window function determination and radiometer spectral noise parametrization. A detailed description of the updated data processing that produces maximum likelihood sky map estimates is presented, along with the methods used to produce reduced resolution maps and corresponding noise covariance matrices. Finally, two methods used to evaluate the noise of the full resolution sky maps are presented along with several representative year-to-year null tests.

Conclusions: The sky maps produced from data from different observational epochs are consistent.

On the night of December 6, 1979, an obscure Stanford Linear Accelerator Center postdoc fellow was up late, sweating over an even more obscure problem about particles called magnetic monopoles. Looking at his calculations the next day, Alan Guth discovered cosmic inflation, an idea which some have later called the most important in cosmology since the big bang. Big bang physics describes how matter and energy developed over the last 13.7 billion years. The concept of inflation poses that the universe expanded many trillion times its size in less than a trillionth of a second at the outset of the big bang. This is now supported by recent findings from the NASA's Wilkinson Microwave Anisotropy Probe (WMAP), and is based on 3 years of continuous observations of the cosmic microwave background, the afterglow light produced when the universe was less than a million years old. WMAP can resolve features in the cosmic microwave background based on polarization (such as sunlight reflecting off of a shiny object), or the way light is changed by the environment through which it passes. One long-held prediction was that the brightness would be the same for features of all sizes. In contrast, inflation predicts the relative brightness decreases as the features get small, a trend seen in the new data.

\section{Prize Winners: the fruit falls not far from the stem}

Our yearbooks have traditionally ended with a prize, and not a single year goes by without important prizes and awards that go to innovations that affect pediatric endocrinology. This year's Albert Lasker Award for Basic Medical Research, the second most prestigious prize after the Nobel, went to Ernest A. McCulloch and James E. Till for ingenious experiments that first identified stem cells. Interestingly, the driving force behind McCullough and Till's research was the realization that radiation from nuclear bombs and or x-ray machines can kill blood cell production, and that this can be rescued in mice exposed to such radiation by injections of bone marrow from healthy mice. It was several years later that this research led to the discovery of local growth factors like GCSF and hormones like erythropoietin. It developed bone marrow transplantation into a quantitative science and technology that sustains life in so many conditions, and eventually also lead to a score of pediatric endocrine complications. In recent years we learnt about embryonic stem cells that may allow us to regenerate damaged body parts, and in our field, it has to do with the promising $\beta$-cell generation from stem cells. Ethical issues were raised, but it seems that we will find ways to make positive use of this ingenious discovery.

References

1. Lyon MF: Gene action in the X-chromosome of the mouse (Mus musculus L.). Nature 1961;190:372-373.

2. Rogers YH, Venter JC: Genomics: massively parallel sequencing. Nature 2005;437:326-327.

3. Adolphs R, Gosselin F, Buchanan TW, Tranel D, Schyns P, Damasio AR: A mechanism for impaired fear recognition after amygdala damage. Nature 2005;433:68-72. 
